\title{
ARTICLE
}

\section{A randomized trial of an NMDA receptor antagonist for reversing corticosteroid effects on the human hippocampus}

\author{
E. Sherwood Brown ${ }^{1}$, Alexandra Kulikova', Erin Van Enkevort ${ }^{1}$, Alyson Nakamura ${ }^{1}{ }^{1}$, Elena I. Ivleva ${ }^{1}$, Nicholas J. Tustison ${ }^{2}$, \\ Jared Roberts ${ }^{2}$, Michael A. Yassa ${ }^{2}$, Changho Choi $^{3}$, Alan Frol ${ }^{1}$, David A. Khan ${ }^{4}$, Miguel Vazquez ${ }^{4}$, Traci Holmes ${ }^{1}$ and Kendra Malone ${ }^{1}$
}

Preclinical and clinical research indicates that excess corticosteroid is associated with adverse effects on the hippocampus. Animal model data suggest that N-methyl-D-aspartate (NMDA) receptor antagonists may block corticosteroid effect on the hippocampus. This translational clinical trial investigated the effect of memantine vs. placebo on hippocampal subfield volume in humans receiving chronic corticosteroid therapy. Men and women $(N=46)$ receiving chronic prescription corticosteroid therapy were randomized to memantine or placebo in a double-blind, crossover design (two 24-week treatment periods, separated by a 4-week washout) for 52 weeks. Structural magnetic resonance imaging was obtained at baseline and after each treatment. Data were analyzed using repeated measures analysis of variance. Mean corticosteroid dose was $7.69 \pm 6.41 \mathrm{mg} /$ day and mean duration $4.90 \pm 5.61$ years. Controlling for baseline volumes, the left DG/CA3 region was significantly larger following memantine than placebo $(p=.011)$. The findings suggest that an NMDA receptor antagonist attenuates corticosteroid effect in the same hippocampal subfields in humans as in animal models. This finding has both mechanistic and clinical implications. Attenuation of the effect of corticosteroids on the human DG/CA3 region implicates the NMDA receptor in human hippocampal volume losses with corticosteroids. In addition, by suggesting a drug class that may, at least in part, block the effects of corticosteroids on the human DG/CA3 subfield, these results may have clinical relevance for people receiving prescription corticosteroids, as well as to those with cortisol elevations due to medical or psychiatric conditions.

Neuropsychopharmacology (2019) 44:2263-2267; https://doi.org/10.1038/s41386-019-0430-8

\section{INTRODUCTION}

An extensive literature in animal models suggests that stress or corticosteroid (glucocorticoid receptor agonists) administration is associated with reduction in hippocampal dendritic length and number [1-4] that appears to be most pronounced in the CA3 region [5]. In animal models, glutamate, in concert with corticosteroids, plays an essential role in stress-induced remodeling of hippocampal neurons, as well as in stressrelated and glucocorticoid-related neurotoxicity [6]; and the glutamate release inhibitor, phenytoin, blocks the effects of exogenous corticosteroids on the hippocampus [7]. The hippocampus has a high concentration of NMDA receptors [8]. NMDA receptors in the hippocampus are critical to memory and synaptic plasticity $[9,10]$. The overactivation of NMDA receptors that is associated with excess glutamate can lead to excitotoxicity, apoptosis and neuronal injury, and death [11-13]. Pretreatment with the competitive NMDA receptor antagonist (CGP 43487) blocked chronic stress-induced atrophy of apical dendrites in the rat hippocampus [14]. In another report, the NMDA receptor antagonist CGP 39551 prevented stress-induced impairment in hippocampal longterm potentiation [15]. Furthermore, the NMDA receptor antagonist memantine improved spatial working memory in acute and repeated stress [16]. Thus, literature from animal models suggests that the NMDA receptor is intimately involved in mediating the effects of corticosteroids on the hippocampus.

Limited human literature also suggests that chronic corticosteroid exposure is associated with a reduction in declarative memory performance and decreased hippocampal volume [17-19]. A small pilot study reported that 8-weeks of therapy with the NMDA receptor antagonist memantine, as compared to placebo, improved declarative memory performance in patients receiving chronic oral corticosteroid therapy [20]. The current report uses state-of-the art structural neuroimaging analysis to examine the effects of 24 weeks of memantine or placebo on hippocampal subfield volumes and memory in patients with medical illnesses receiving chronic prescription corticosteroid therapy. The objective of this study was to translate the preclinical literature on the ability of NMDA receptor antagonists to block the effects of corticosteroids on the hippocampus to a human clinical population. The hypothesis was that memantine would, at least partially, reverse the effects of corticosteroids on hippocampal subfield volume and memory in humans.

\footnotetext{
'Department of Psychiatry, The University of Texas Southwestern Medical Center, Dallas, TX 75390, USA; ${ }^{2}$ Department of Neurobiology and Behavior, Center for the Neurobiology of Learning and Memory, University of California at Irvine, Irvine, CA 92697, USA; ${ }^{3}$ Departments of Radiology and the Advanced Imaging Research Center, The University of Texas Southwestern Medical Center, Dallas, TX 75390, USA and ${ }^{4}$ Department of Internal Medicine, The University of Texas Southwestern Medical Center, Dallas, TX 75390, USA Correspondence: E Sherwood Brown (Sherwood.Brown@UTSouthwestern.edu) Deceased: Jared Roberts
}

Received: 7 January 2019 Revised: 10 May 2019 Accepted: 1 June 2019 Published online: 10 June 2019 


\section{MATERIALS AND METHODS}

Medically stable outpatients receiving chronic oral corticosteroid therapy were enrolled in a 52-week randomized, double-blind, placebo-controlled, crossover, trial of memantine between 5/18/ 2012 and 1/15/2016 (NCT01656187) at UT Southwestern Medical Center, Dallas, TX, USA. The study was approved by the UT Southwestern Institutional Review Board (IRB). Participants provided IRB-approved written informed consent and were primarily recruited from Parkland Hospital Chronic Kidney Disease Clinic, Asthma and Allergy Clinic, and UT Southwestern St. Paul's Pulmonary Clinic. A structured clinical interview for DSM 4 (SCID-CV) was administered at baseline to assess psychiatric diagnoses that were exclusionary.

At the randomization visit, memantine or identical placebo (Abrams Royal Compounding Pharmacy, Dallas, TX, USA) was initiated at $5 \mathrm{mg} /$ day, increased to $5 \mathrm{mg} \mathrm{BID}$ at week 2, $10 \mathrm{mg} \mathrm{AM}$ and $5 \mathrm{mg}$ HS at week 3 , and $10 \mathrm{mg}$ BID weeks 4-24. Participants received treatment with both memantine and placebo for 24 weeks in a randomized crossover design. Thus, a participant might receive memantine first (weeks $0-24$ ) or second (weeks 28-52). There was always a washout period with no study drug administration from weeks 24-28. The order of treatment was randomized (1:1) using a random number generator by a statistician with no participant contact, and the randomization was stratified by the baseline dose of prednisone ( $<20 \mathrm{mg} /$ day vs. $\geq 20 \mathrm{mg}$ /day). Structural MRI was obtained at baseline, week 24 and week 52, following the treatment with memantine or placebo. The Hopkins Verbal Learning Test-Revised (HVLT-R) assessed declarative memory every four weeks. The HVLT-R consists of 12 nouns read aloud for five consecutive trials with each trial followed by a free-recall trial. Four comparable alternate forms, given in a set order, were used to minimize practice effects. All study personnel involved in participant assessment were blinded to the treatment conditions.

Included were men and women age 18-65 years receiving prednisone therapy of at least $5 \mathrm{mg}$ per day for $\geq 6$ months with anticipated treatment for $\geq 12$ additional months. Excluded were people with illnesses associated with CNS involvement (e.g., seizures, brain tumors, head injury with loss of consciousness) or cognitive impairment (e.g., substance dependence within 2 years, mood disorders, psychotic disorders), vulnerable populations (e.g., severe cognitive impairment, pregnant or nursing women, prisoners), contraindications to memantine therapy (e.g., severe side effects in the past), danger to self or others as defined by $>1$ lifetime suicide attempt or assault, any suicide attempt or assault within the past year, and active suicidal or homicidal ideation with plan and intent or metal implants, claustrophobia, or other contraindications to MRI. Potential participants with mood symptoms secondary to corticosteroids (based on SCID) were not excluded because this could selectively exclude subjects who are sensitive to the CNS effects of corticosteroids,

\section{MR methods}

Neuroimaging was performed using a whole-body horizontal bore Philips 3T scanner (Philips Medical Systems; Best, The Netherlands) at the Advanced Imaging Research Center, UT Southwestern Medical Center. The scanner had an integrated body coil for radiofrequency (RF) transmission and an 8-channel phased-array coil for signal reception. Following a survey scan, sagittal $\mathrm{T}_{1}$-weighted images of the brain (MP-RAGE: TE/TI/TR $=3.8 / 875 / 1360 \mathrm{~ms}, 256 \times$ $256 \times 160 \mathrm{~mm}^{3}$ field of view, 160 slices, voxel size $1 \times 1 \times 1 \mathrm{~mm}^{3}$ ). MP-RAGE images were subsequently used for hippocampal voxel positioning for MRS, as well as hippocampal subfield segmentation.

Structural MRI volumetric analysis

Hippocampal subfield segmentation was performed using a consensus labeling approach based on a set of 19 T2-weighted images acquired using an optimized hippocampus-specific acquisition protocol (image resolution: $0.47 \times 0.47 \mathrm{~mm}^{2}$ in-plane, $2.0 \mathrm{~mm}$ slice thickness) from cognitively normal volunteers who were manually labeled using a highly reliable anatomical protocol used in prior published work for hippocampal subfields [21-24]. Anatomical labeling of the atlas set comprised separate labels for right and left dentate gyrus (DG)/CA3, $C A 1$, and subiculum. Scans were coupled with corresponding T1-weighted images (image resolution: $0.75 \times 0.75 \times 0.75 \mathrm{~mm}^{3}$ ) which were acquired for multi-spectral atlas-based registration. To propagate a weighted consensus labeling from an expertly labeled atlas set to the unlabeled T1-weighted images of our study cohort, we spatially normalized the atlas set to the unlabeled subject and applied the joint label fusion technique. Advanced Normalization Tools (ANTs) package was used for both spatial normalization [25] and consensus-based labeling (i.e., joint label fusion) [26]. First, the intra-subject atlas T1/T2 rigid transforms were calculated. To minimize total number of deformable registrations, a "pseudo-geodesic" approach to data alignment was used [27]. This required construction of an optimal T1-weighted template [28] representing the average shape/intensity information of the T1 component of the atlas set. Deformable transformations between each T1-weighted image of the study cohort and the T1 atlas template were calculated. Transformation between the atlas labels and unlabeled study cohort image was then computed by concatenating the $\mathrm{T} 1$ atlas $/ \mathrm{T} 2_{\text {atlas }}$ rigid transformation, the $\mathrm{T} 1_{\text {atlas }} / \mathrm{T} 1$ template deformable transformation, and the $\mathrm{T} 1$ template/and $\mathrm{T} 1_{\text {subject }}$ deformable transforms. Once the atlas set was normalized to the unlabeled participant, regional labeling was determined using weighted averaging where the weighting takes into account the unique intensity information contributed by each atlas member. After visual quality assessment to confirm the output of the labeling procedures, voxels within the labeled regions were counted and multiplied by the voxel resolution to calculate volume in cubic millimeters.

\section{Statistical analysis}

Power analysis of the cognitive measures was based on the effect size observed on the HVLT-R in our pilot study [20]. A repeated measures approach was used to analyze the data to account for the within-subjects cross-over design. Structural MRI data were collected at baseline and after each 24-week treatment period. While controlling for baseline data for the variable of interest, a one-way repeated measures ANCOVA with medication included as a within-subjects factor was used to examine the influence of treatment on hippocampal volume at 24 and 52 weeks. A total of 32 participants had week 52 data. However, these study completers, in some cases, had missing data at some assessment points (e.g., missing MRI data). Because neuroimaging was only obtained at baseline and at weeks 24 and 52 these data were analyzed on a completer sample with neuroimaging data at baseline, week 24 and week $52(N=26)$. To assess declarative memory, a 2 (treatment) by 2 (time point) ANOVA was utilized. Specifically, medication (placebo vs. memantine) and time point (pre medication vs. post medication) were included in the model as within-subject variables. Because the declarative memory data were collected at four week intervals, we included all participants with at least one assessment from both treatment conditions $(N=32)$ in the analysis. Differences within the 24-week treatment period, as well as between treatments were examined. Because of the within-subject crossover design, demographic characteristics were not controlled for in the analysis. The Benjamini-Hochberg procedure was used to control for false discovery rate (the proportion of significant results that are false positives) from examination of multiple hippocampal subfields $[29,30]$. 


\section{RESULTS}

Forty-six $(N=46)$ participants were randomized and received memantine and placebo, with 26 participants completing the study. Baseline characteristics of participants are provided in Table 1. The study included 19 men and 27 women. The participants were racially and ethnically diverse. The majority of participants were receiving chronic corticosteroid therapy to prevent renal transplant rejection. The mean dose of corticosteroid therapy at baseline was $7.6 \pm 6.4 \mathrm{mg} /$ day and the mean duration was $58.8 \pm 67.3$ months. The mean age of the participants was 43 years.

Structural MRI analyses examined three hippocampal subfields: DG/CA3, CA1, and subiculum. Table 2 presents the means, standard deviations, and $p$-values of the hippocampal subfields after placebo and memantine conditions. Figure 1 illustrates the three hippocampal subfields (CA1, DG/CA3, and subiculum) in a

\begin{tabular}{|c|c|c|}
\hline \multicolumn{3}{|l|}{ Demographics $(N=46)$} \\
\hline \multicolumn{3}{|l|}{ Characteristics } \\
\hline & M & $S D$ \\
\hline Age & 43.18 & 12.42 \\
\hline Education (years) & 13.50 & 1.97 \\
\hline Corticosteroid dose (mg) & 7.59 & 6.41 \\
\hline \multirow[t]{2}{*}{ Corticosteroid use (years) } & 4.90 & 5.61 \\
\hline & $N$ & $\%$ \\
\hline \multicolumn{3}{|l|}{ Sex } \\
\hline Male & 27 & $58.7 \%$ \\
\hline Female & 19 & $41.3 \%$ \\
\hline \multicolumn{3}{|l|}{ Race/Ethnicity } \\
\hline African American & 26 & $56.5 \%$ \\
\hline Caucasian & 4 & $8.7 \%$ \\
\hline Hispanic & 16 & $34.8 \%$ \\
\hline \multicolumn{3}{|l|}{ Marital status } \\
\hline Married/cohabitating & 15 & $32.6 \%$ \\
\hline Divorced/widowed & 6 & $13.0 \%$ \\
\hline Never married & 25 & $54.3 \%$ \\
\hline \multicolumn{3}{|c|}{ Medical diagnosis requiring corticosteroids } \\
\hline Kidney transplant & 32 & $69.6 \%$ \\
\hline Systemic lupus erythematosus & 11 & $23.9 \%$ \\
\hline Rheumatoid arthritis & 2 & $4.3 \%$ \\
\hline Asthma & 1 & $2.2 \%$ \\
\hline
\end{tabular}

Table 2. Difference in hippocampal subfields following memantine vs. placebo treatment

\begin{tabular}{|c|c|c|c|c|c|}
\hline \multirow[t]{2}{*}{ ROI } & \multicolumn{2}{|l|}{ Placebo } & \multicolumn{2}{|c|}{ Memantine } & \multirow[t]{2}{*}{$p$-value } \\
\hline & $M$ & SD & $M$ & SD & \\
\hline Left DG/CA3 & 607.90 & 113.12 & 619.58 & 106.98 & .011 \\
\hline Right DG/CA3 & 646.12 & 155.45 & 668.46 & 129.51 & .676 \\
\hline Left CA1 & 1654.01 & 210.90 & 1642.88 & 213.89 & .523 \\
\hline Right CA1 & 1615.77 & 220.09 & 1616.31 & 245.88 & .055 \\
\hline Left subiculum & 782.18 & 152.65 & 779.31 & 134.99 & .351 \\
\hline Right subiculum & 783.15 & 145.57 & 798.42 & 114.42 & .764 \\
\hline
\end{tabular}

representative participant. There was no overall effect of time on any regional brain volume ( $p$-values all >0.05), except for the volume of the left CA1 $(p=.010)$. Pairwise comparisons showed that volume decreased significantly from baseline to week 52 $(p=.031)$ and from week 24 to week $52(p=.003)$, but not between baseline and week $24(p=.780)$. However, an effect of treatment condition was observed. Controlling for baseline subfield volume, the left DG/CA3 region was significantly larger ( 2\%) following 24 weeks of memantine as compared to placebo $(p=.011)$ which was significant using a false discovery rate (Q) of 0.1 with the Benjamini-Hochberg procedure. A trend in the same direction was observed with the right CA1 subfield $(p=.055)$. A post hoc analysis, using paired $t$-test, examined changes in brain volumes among participants who received placebo first $(N=20)$. No significant change in volume of any of the brain regions was observed ( $p$ values all $>.05$ )

Declarative memory, as assessed by HVLT-R Total $T$ scores, did not differ significantly between the memantine and placebo (mean [ \pm SE] Baseline to Week $24 ; 39.40 \pm 1.82$ to $37.67 \pm 1.61$ vs. $36.93 \pm 1.72$ to $38.37 \pm 1.92, p=.376$ ). Changes in HVLT-R scores did not correlate significantly with changes in brain volumes in the memantine or placebo conditions ( $P$ values $>0.05$ ).

Due to complex medical history of the study participants, different adverse events that were determined to be unrelated to the medication were reported throughout the study. During the treatment with memantine, the only discontinuation was from a participant who became pregnant while in the study. Other adverse events included one ear infection, one diagnosis of Clostridium difficile infection, one tooth fracture, one Fournier's gangrene diagnosis, two respiratory exacerbations (asthma flare and pneumonia), one report of abnormal stool color, one reported concussion due to a motor vehicle accident, two reports of elevated blood pressure and one report of low blood pressure. During the treatment with placebo, one participant discontinued the study due to constipation and one patient discontinued after she received a diagnosis of vulvar squamous cell carcinoma for which the patient underwent a laser ablation procedure. Other reported adverse events included one episode of chest pains, one episode of hypokalemia that resulted in an emergency room visit, one report of a herpes zoster outbreak, one lumbar spine injury due to a motor vehicle accident, one episode of gout flare, one episode of right shoulder pain, one finding of a benign ovarian cyst, two urinary tract infections, and one lupus flare. These events were determined to not be related to the study or the study medication.

Memantine was well tolerated. The number of potential side effects that were determined to be definitely or possibly related to the study medication were not significantly different between memantine and placebo conditions $\left(x^{2}(9)=9.22, p=.420\right)$. Of those who reported side effects, four participants reported more than one side effect. A total of 16 side effects were reported, with the majority (69\%) were related to gastrointestinal problems (e.g., bloating, constipation, diarrhea, nausea) in both treatment conditions. No IRB-reportable adverse events occurred. A total of $89.5 \%$ reported greater than $80 \%$ study adherence by pill counts with memantine as compared to $95.8 \%$ with placebo $(x 2(1)=.661, p=.416)$.

\section{DISCUSSION}

The literature suggests that corticosteroids are associated with changes in dendritic length in the CA3 region of the hippocampus [5]. In animal models, these changes can be prevented by pretreatment with an NMDA receptor antagonist [14]. This is, to our knowledge, the first neuroimaging study to examine whether an NMDA receptor antagonist attenuates the effects of corticosteroids on the human hippocampus. Therefore, the current report extends preclinical findings to humans. The findings suggest that 


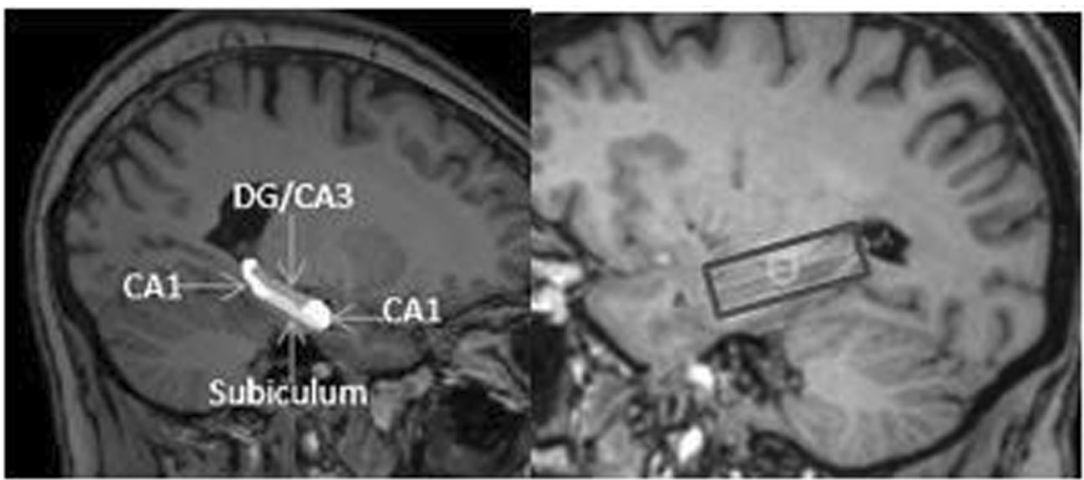

Fig. 1 Representative images of hippocampal segmentation (left) and voxel positioning (right)

memantine administration was associated with a significantly larger CA3/DG ( 2\% difference) than placebo in people receiving medically necessary chronic prescription corticosteroid therapy. A trend in the same direction was observed in the CA1 region.

These MRI findings have both mechanistic and clinical significance. First, the findings suggest that an NMDA receptor antagonist, such as memantine, attenuates the effects of corticosteroids in the same hippocampal subregion in humans as in animals. It is not, however, possible to determine in humans whether the changes are related to reversal of dendritic shortening, changes in overall neuronal size or number, changes in fluid, or other mechanisms. Second, the findings may have clinical significance for people treated with prescription corticosteroids, or with cortisol elevations due to Cushing's Disease or even mood disorders. Prior research suggests that people receiving chronic prescription corticosteroid therapy have total mean hippocampal volumes that are about $8-9 \%$ smaller than people with similar medical histories not receiving corticosteroids [19]. Memantine administration over a period of 24 weeks appeared to partially reverse some of hippocampal atrophy in this population. In adults, an annual reduction in hippocampal volume of about $0.82 \%$ per year is reported [31]. Thus, 24 weeks of memantine therapy may have reversed, on average, about two years of normal atrophy.

The study did not observe a significant improvement in declarative memory as assessed by the HVLT-R. At least in healthy controls, the relationship between hippocampal volume and memory is weak [32]. Our prior research has not found statistically significant relationships between declarative memory performance and hippocampal volume in corticosteroid-treated patients $[19,33]$. In the present study, the HVLT was used because multiple equivalent versions are available, which was needed for a longitudinal study. However, the HVLT-R only has 12 words, as opposed to 15 in other word lists tests. A report suggested that the HVLT-R was less sensitive to subtle cognitive changes than the CVLT [34]. Thus, we may have not been able to detect small cognitive changes over a relatively brief period of time with this instrument. Alternatively, the changes observed in the hippocampal subfield volume may be an earlier and more sensitive biomarker of the ability of memantine to reverse the effects of corticosteroids on the hippocampus compared to memory testing.

The study has limitations. While the within-subject crossover design has many advantages in a neuroimaging study and has greater statistical power than an analysis of a parallel-group sample of the same size, carryover effects are possible. However, the very long four-week washout period was about 10 times the half-life of memantine. While large for a study of this nature, this sample size was not sufficient to detect subtle effects of memantine on memory or the hippocampus. The attrition rate is also a limitation. Only $32 / 46(70 \%)$ of the participants enrolled completed the study and some of these had missing data. Therefore, we only had MRI data on 26 participants. Since memantine appeared to be well-tolerated the relatively high attrition rate may reflect the length of the study (one year) and the medically ill patient population. Strengths of the study include the double-blind, placebo-controlled design and the state-of-the art hippocampal subfield imaging analysis. To our knowledge, no published research studies have examined hippocampal subfields in corticosteroid-treated patients.

In conclusion, memantine was associated with a larger CA3/DG volume than placebo when given to corticosteroid-treated patients. The findings translate preclinical research on the ability of NMDA receptor antagonists to block the effects of corticosteroids on the hippocampus and highlight the importance of the NMDA receptor in mediating the effects of corticosteroids on the hippocampus. Furthermore, the findings suggest memantine may have potential as a neuroprotective agent in people receiving prescription corticosteroids therapy or with elevated levels of endogenous cortisol.

\section{FUNDING AND DISCLOSURE}

This work was supported by NIDA grant R01 DA029596. Dr. Brown has ongoing or recent research funding from NIAAA, NIA, NHLBI, $\mathrm{NCClH}$ and Stanley Medical Research Institute, and Otsuka Pharmaceuticals and serves on a an advisory board for Allergan. Dr. Ell has ongoing support from NIMH. Dr. MAY is supported by NIMH R01MH102392 and NIA R01AG053555. The authors declare no competing interests.

\section{ADDITIONAL INFORMATION}

Supplementary Information accompanies this paper at (https://doi.org/10.1038/ s41386-019-0430-8).

Publisher's note: Springer Nature remains neutral with regard to jurisdictional claims in published maps and institutional affiliations.

\section{REFERENCES}

1. Magarinos AM, Verdugo JM, McEwen BS. Chronic stress alters synaptic terminal structure in hippocampus. Proc Natl Acad Sci USA. 1997;94:14002-8.

2. Vyas A, Mitra R, Shankaranarayana Rao BS, Chattarji S. Chronic stress induces contrasting patterns of dendritic remodeling in hippocampal and amygdaloid neurons. J Neurosci. 2002;22:6810-8.

3. Wood GE, Young LT, Reagan LP, Chen B, McEwen BS. Stress-induced structural remodeling in hippocampus: prevention by lithium treatment. Proc Natl Acad Sci USA. 2004;101:3973-8.

4. Cameron HA, Schoenfeld TJ. Behavioral and structural adaptations to stress. Front Neuroendocrinol. 2018;49:106-13.

5. McEwen BS. Stress-induced remodeling of hippocampal CA3 pyramidal neurons. Brain Res. 2016;1645:50-4.

6. Popoli M, Yan Z, McEwen BS, Sanacora G. The stressed synapse: the impact of stress and glucocorticoids on glutamate transmission. Nat Rev Neurosci. 2012;13:22-37. 
7. Magarinos AM, McEwen BS, Flugge G, Fuchs E. Chronic psychosocial stress causes apical dendritic atrophy of hippocampal CA3 pyramidal neurons in subordinate tree shrews. J Neurosci. 1996;16:3534-40.

8. Monaghan DT, Cotman CW. Distribution of N-methyl-D-aspartate-sensitive L-[3H] glutamate-binding sites in rat brain. J Neurosci. 1985;5:2909-19.

9. Baez MV, Cercato MC, Jerusalinsky DA. NMDA receptor subunits change after synaptic plasticity induction and learning and memory acquisition. Neural Plast. 2018;2018:5093048.

10. Traynelis SF, Wollmuth LP, McBain CJ, Menniti FS, Vance KM, Ogden KK, et al. Glutamate receptor ion channels: structure, regulation, and function. Pharm Rev. 2010;62:405-96.

11. Tackenberg C, Grinschgl S, Trutzel A, Santuccione AC, Frey MC, Konietzko U, et al NMDA receptor subunit composition determines beta-amyloid-induced neurodegeneration and synaptic loss. Cell Death Dis. 2013;4:e608.

12. Benarroch EE. NMDA receptors: recent insights and clinical correlations. Neurology. 2011;76:1750-7.

13. Dong $Y$, Kalueff AV, Song C. N-methyl-d-aspartate receptor-mediated calcium overload and endoplasmic reticulum stress are involved in interleukin-1beta-induced neuronal apoptosis in rat hippocampus. J Neuroimmunol. 2017;307:7-13.

14. Magarinos AM, McEwen BS. Stress-induced atrophy of apical dendrites of hippocampal CA3c neurons: involvement of glucocorticoid secretion and excitatory amino acid receptors. Neuroscience. 1995;69:89-98.

15. Kim JJ, Foy MR, Thompson RF. Behavioral stress modifies hippocampal plasticity through N-methyl-D-aspartate receptor activation. Proc Natl Acad Sci USA. 1996;93:4750-3.

16. Amin SN, El-Aidi AA, Ali MM, Attia YM, Rashed LA. Modification of hippocampal markers of synaptic plasticity by memantine in animal models of acute and repeated restraint stress: implications for memory and behavior. Neuromolecular Med. 2015;17:121-36.

17. Keenan PA, Jacobson MW, Soleymani RM, Mayes MD, Stress ME, Yaldoo DT. The effect on memory of chronic prednisone treatment in patients with systemic disease. Neurology. 1996;47:1396-402.

18. Wilner AP, de Varennes B, Gregoire PA, Lupien S, Pruessner JC. Glucocorticoids and hippocampal atrophy after heart transplantation. Ann Thorac Surg. 2002;73:1965-7.

19. Brown ES, Woolston JD, Frol A, Bobadilla L, Khan DA, Hanczyc $M$, et al Hippocampal volume, spectroscopy, cognition, and mood in patients receiving corticosteroid therapy. Biol Psychiatry. 2004;55:538-45.
20. Brown ES, Vazquez M, Nakamura A. Randomized, placebo-controlled, crossover trial of memantine for cognitive changes with corticosteroid therapy. Biol Psychiatry. 2008;64:727-9.

21. Yassa MA, Stark CE. A quantitative evaluation of cross-participant registration techniques for MRI studies of the medial temporal lobe. Neuroimage. 2009;44:319-27.

22. Yassa MA, Stark CE. Pattern separation in the hippocampus. Trends Neurosci. 2011;34:515-25.

23. Yassa MA, Stark SM, Bakker A, Albert MS, Gallagher M, Stark CE. High-resolution structural and functional MRI of hippocampal CA3 and dentate gyrus in patients with amnestic Mild Cognitive Impairment. Neuroimage. 2010;51:1242-52.

24. Yassa MA, Mattfeld AT, Stark SM, Stark CE. Age-related memory deficits linked to circuit-specific disruptions in the hippocampus. Proc Natl Acad Sci USA 2011;108:8873-8.

25. Avants BB, Tustison NJ, Song G, Cook PA, Klein A, Gee JC. A reproducible evaluation of ANTs similarity metric performance in brain image registration. Neuroimage. 2011;54:2033-44.

26. Wang H, Suh JW, Das SR, Pluta JB, Craige C, Yushkevich PA. Multi-atlas segmentation with joint label fusion. IEEE Trans pattern Anal Mach Intell. 2013;35:611-23.

27. Tustison NJ, Avants BB. Explicit B-spline regularization in diffeomorphic image registration. Front Neuroinform. 2013;7:39.

28. Avants BB, Yushkevich P, Pluta J, Minkoff D, Korczykowski M, Detre J, et al. The optimal template effect in hippocampus studies of diseased populations. Neuroimage. 2010;49:2457-66.

29. Benjamini Y, Hochberg Y. Controlling the false discovery rate: a practical and powerful approach to multiple testing. J R Stat Soc Ser B. 1995;57:289-300.

30. Simes RJ. An improved Bonferroni procedure for multiple tests of significance. Biometrika. 1986;73:751-4.

31. Scahill RI, Frost C, Jenkins R, Whitwell JL, Rossor MN, Fox NC. A longitudinal study of brain volume changes in normal aging using serial registered magnetic resonance imaging. Arch Neurol. 2003;60:989-94.

32. Van Petten C. Relationship between hippocampal volume and memory ability in healthy individuals across the lifespan: review and meta-analysis. Neuropsychologia. 2004;42:1394-413.

33. Brown ES, Jeon-Slaughter $\mathrm{H}$, Lu H, Jamadar R, Issac S, Shad M, et al. Hippocampal volume in healthy controls given 3-day stress doses of hydrocortisone. Neuropsychopharmacology. 2015;40:1216-21.

34. Lacritz LH, Cullum CM. The Hopkins verbal learning test and CVLT: a preliminary comparison. Arch Clin Neuropsychol. 1998;13:623-8. 